\title{
Neuronal transcriptional networks in life span control
}

\author{
A.V. Symonenko ${ }^{1}$, N.V. Roshina ${ }^{1,2}$, A.V. Krementsova ${ }^{1,3}$, E.R. Veselkina ${ }^{1}$, \\ O.Y. Rybina ${ }^{1,4}$, E.G. Pasyukova ${ }^{1 *}$ \\ ${ }^{1}$ Institute of Molecular Genetics of RAS, Moscow, Russia \\ ${ }^{2}$ Vavilov Institute of General Genetics of RAS, Moscow, Russia \\ ${ }^{3}$ Emmanuel Institute of Biochemical Physics of RAS, Moscow, Russia \\ ${ }^{4}$ Moscow State Pedagogical University, Institute of Biology and Chemistry, Moscow, Russia \\ *e-mail:egpas@rambler.ru
}

Key words: Life span, transcription factors, the nervous system, Drosophila

Motivation and Aim: The nervous system has long been suggested as a key tissue which defines life span. Numerous and diverse interactions between the nervous system and life span are reciprocal and intimately linked. Several pathways play key roles both in longevity control and in shaping the nervous system during development. The question remains whether their impact on lifespan is related to their developmental functions, or whether it is explained by other pleiotropic influences later in life.

To get closer to solving this issue, we assess the effects of the genes encoding neuronal transcription factors on life span using genetic approaches combined with RT-qPCR, RNA-seq and immunohistochemical analyses.

Results: We previously demonstrated that mutations/reversions and tissue-specific RNAi knockdown and overexpression of several genes encoding transcription factors involved in the development of the nervous system (stc, Lim3, and others) affect Drosophila melanogaster life span. Interestingly, embryonic knockdown of both stc and Lim3 increased life spans in adult flies, indicating that it is a decrease in transcription at the developmental stages that matters for longevity. These results made us assume that the long-term effects of alterations in embryonic gene expression may be epigenetically inherited or may affect transcriptional cascades that predetermine properties of the adult. Unexpectedly, alterations in stc and Lim 3 expression which either decreased or increased life span were associated with reduced synaptic function. These preliminary results indicated that the effects of stc and $\operatorname{Lim} 3$ on life span and the development of the nervous system might be uncoupled. To better understand mechanisms providing stc and Lim3 impact on development and aging, we assessed effects of these transcription factors on mRNA levels of possible primary and secondary target genes in embryos. Our results show that stc and Lim3 affect neuronal development, protein metabolism, mitochondrial function and energy homeostasis, which indicates probable molecular mechanisms providing their impact on longevity.

Naturally occurring polymorphisms located in target sites of regulatory proteins in the regulatory regions of stc and $\operatorname{Lim} 3$ were associated with variation in life span and gene transcription. Effects of naturally occurring variation on gene transcription were directly confirmed in experiments with cell culture and flies. X-ChIP technique was used to show that Polycomb/Trithorax response element was located in the Lim3 distal promoter and ensured physical and functional interactions with the Polycomb group (PcG) proteins. Based on these findings, we suggested that PcG proteins are directly involved in the regulation of $\operatorname{Lim} 3$ transcription and might be important for lifespan control.

Conclusion: Systemic regulation of neuronal transcriptional networks is proposed as one of the mechanisms regulating life span.

Acknowledgements: Supported by the RFBR grant No. 18-04-01127-a and Russian State Project (01201355486). 